ARTICLE

Received 23 Jul 2013 | Accepted 12 Dec 2013 | Published 17 Jan $2014 \quad$ DOl: 10.1038/ncomms4093

\title{
Ultrathin rhodium nanosheets
}

\author{
Haohong Duan ${ }^{1}$ Ning Yan ${ }^{2}$, Rong Yu ${ }^{3}$, Chun-Ran Chang ${ }^{1}$, Gang Zhou ${ }^{4}$, Han-Shi Hu${ }^{1}$, Hongpan Rong ${ }^{1}$, \\ Zhiqiang Niu', Junjie Mao ${ }^{1}$, Hiroyuki Asakura ${ }^{5}$, Tsunehiro Tanaka ${ }^{6,7}$, Paul Joseph Dyson ${ }^{8}$, Jun $\mathrm{Li}^{1}$ \\ $\&$ Yadong $\mathrm{Li}^{1}$
}

Despite significant advances in the fabrication and applications of graphene-like materials, it remains a challenge to prepare single-layered metallic materials, which have great potential applications in physics, chemistry and material science. Here we report the fabrication of poly(vinylpyrrolidone)-supported single-layered rhodium nanosheets using a facile solvothermal method. Atomic force microscope shows that the thickness of a rhodium nanosheet is $<4 \AA$. Electron diffraction and $X$-ray absorption spectroscopy measurements suggest that the rhodium nanosheets are composed of planar single-atom-layered sheets of rhodium. Density functional theory studies reveal that the single-layered Rh nanosheet involves a $\delta$-bonding framework, which stabilizes the single-layered structure together with the poly(vinylpyrrolidone) ligands. The poly(vinylpyrrolidone)-supported single-layered rhodium nanosheet represents a class of metallic two-dimensional structures that might inspire further fundamental advances in physics, chemistry and material science.

\footnotetext{
${ }^{1}$ Department of Chemistry, Tsinghua University, Beijing 100084, China. ${ }^{2}$ Department of Chemical and Biomolecular Engineering, National University of Singapore, 117576 Singapore, Singapore. ${ }^{3}$ Beijing National Center for Electron Microscopy and Laboratory of Advanced Materials, Department of Materials Science and Engineering, Tsinghua University, Beijing 100084, China. ${ }^{4}$ Department of Physics, Tsinghua University, Beijing 100084, China. ${ }^{5}$ Synchrotron Radiation Research Center, Nagoya University, Chikusa-ku, Nagoya 464-8603, Japan. ${ }^{6}$ Department of Molecular Engineering, Kyoto University,

Kyoto 615-8510, Japan. ${ }^{7}$ Unit of Elements Strategy Initiative for Catalysis and Batteries, Kyoto University, Kyoto 615-8510, Japan. ${ }^{8}$ Institut des Sciences et Ingénierie Chimiques, Ecole Polytechnique Fédérale de Lausanne (EPFL), CH-1015 Lausanne, Switzerland. Correspondence and requests for materials should be addressed to Y.L. (email: ydli@mail.tsinghua.edu.cn) or to J.L. (email: junli@mail.tsinghua.edu.cn).
} 
$\mathrm{T}$ he discovery of ultrathin materials composed of single or few atomic layer(s), such as graphene ${ }^{1}$, molybdenum disulphide and boron nitride ${ }^{2}$, has aroused considerable recent interest. Such ultrathin materials exhibit fascinating properties such as high electron mobility, quantum Hall effects, extraordinary thermal conduction, magnetic resonant mode and superconductivity ${ }^{3-8}$. So far, the preparation of ultrathin materials with few atomic layers mainly relies on the material itself having a lamellar structure ${ }^{9}$, a feature that ensures the stability of their few- or even single-layer structures, because of the strong intra-layer chemical bonding and weak inter-layer interaction. In contrast, metal atoms have a strong preference for three-dimensional (3D) close-packed structures. Therefore, ultrathin metallic structures with a plethora of unsaturated atoms are difficult to stabilize and their synthesis remains challenging. More recently, the preparation of surfactant-capped Pd (ref. 10), $\mathrm{Rh}$ (ref. 11), and $\mathrm{Ru}$ nanoplates ${ }^{12}$ with metal thicknesses of $1.8 \mathrm{~nm}, 1.3 \mathrm{~nm}$ and $1.5 \mathrm{~nm}$, respectively, has been reported.

In this work, we report the fabrication of poly(vinylpyrrolidone) (PVP)-supported single-layered rhodium nanosheets (Rh NSs) using a facile solvothermal method. We use aberrationcorrected electronic microscopy, atomic force microscope (AFM) and X-ray absorption spectroscopy (XAS) to reveal that the Rh NSs are composed of planar single-atom-layer sheets of Rh. Density functional theory (DFT) investigations indicate that the single-layered Rh NS possesses a unique $\delta$-bonding framework constructed from metallic $d_{z^{2}}-s$ hybrid orbitals, which stabilizes the single-layered structure together with the PVP ligands.
The discovery of single-layered Rh NS points to the possibility of fabricating similar structures for other metals. Further investigations of such materials might lead to vital advances in physics, chemistry and material science.

\section{Results}

Synthesis and characterizations. Herein, using a solvothermal synthetic approach we obtained semi-transparent Rh NSs with an edge length of $c a .500-600 \mathrm{~nm}$ in the presence of PVP in high yield (see Fig. 1a,b, and Methods for details). These nanosheets are highly sensitive to electron beam irradiation in highresolution transmission electron microscopy (HRTEM) (see Supplementary Fig. 1), suggesting an ultrathin $\mathrm{Rh}$ structure. Moreover, X-ray diffraction pattern of the sample showed no diffraction peaks (see Supplementary Fig. 2), also indicative of an ultrathin nature. Energy-dispersive X-ray studies (see Supplementary Fig. 3) indicate that the individual NSs are composed of Rh NSs capped with PVP, which is further verified by Fourier transform infrared spectroscopy (FTIR, Supplementary Fig. 4).

Structural determination. In order to minimize irradiation damage to the PVP-capped Rh NSs during electron microscopy characterization, low electron exposures were employed with concomitant lower signal-to-noise ratio than that observed under standard imaging conditions. Aberration-corrected electron microscopy with optimum focus was used to improve the
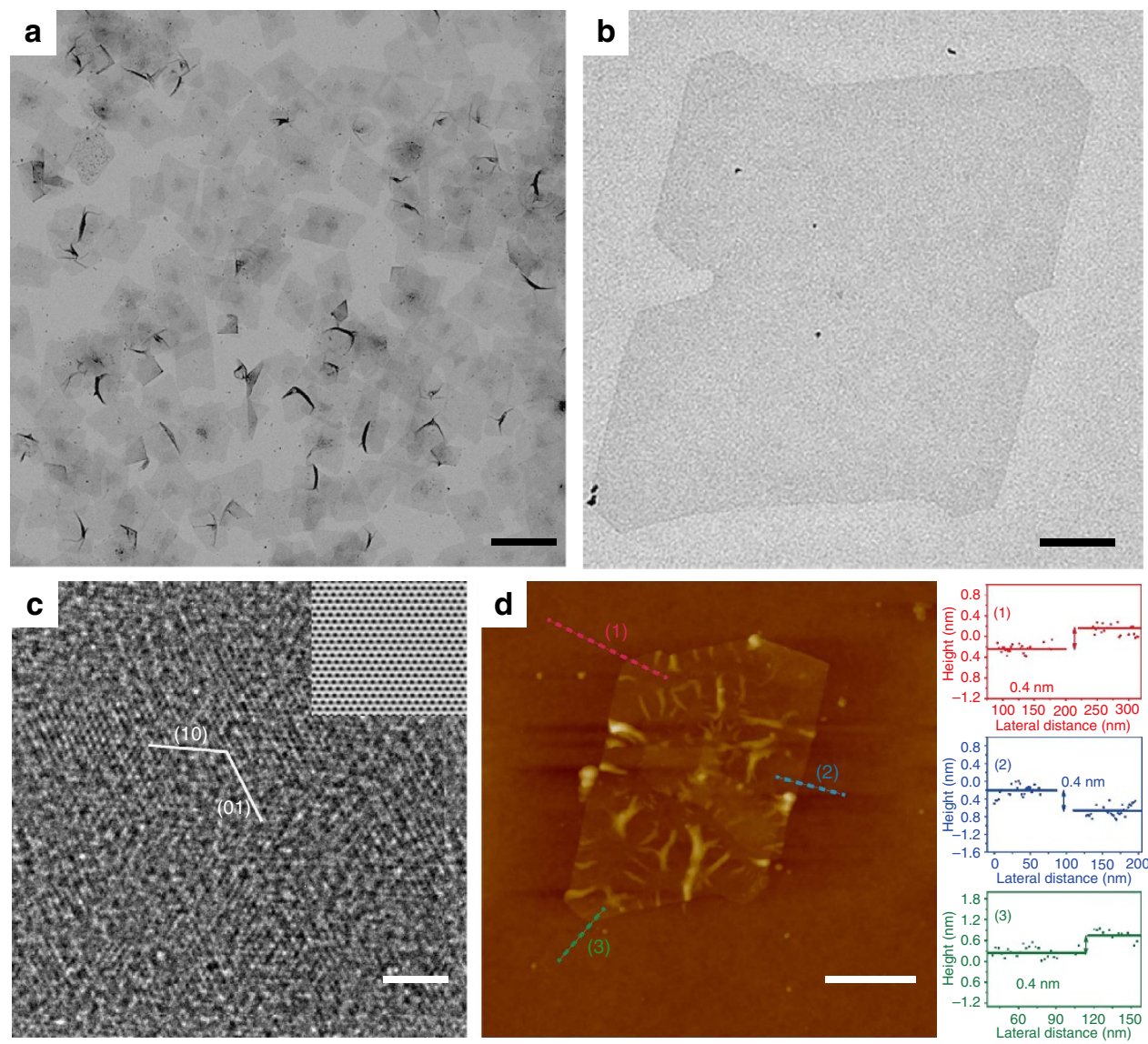

Figure 1 | Characterization of ultrathin Rh NSs. (a) Low-magnification TEM image of the PVP-capped Rh NSs. Scale bar, $1 \mu \mathrm{m}$. (b) High-magnification TEM image of the PVP-capped Rh NSs. Scale bar, $100 \mathrm{~nm}$. (c) Aberration-corrected microscopy image of a PVP-capped Rh NS (inset, the corresponding filtered image using the crystallographic average method to improve signal-to-noise ratio). Scale bar, $2 \mathrm{~nm}$. (d) AFM image and the corresponding height profiles of a bare Rh NS. Scale bar, $200 \mathrm{~nm}$. 
signal-to-noise ratio (see Methods for details). The object plane of the objective lens was set to the mid-plane of the carbon support to reduce the noise from the support ${ }^{13}$. Accordingly, the carbon support was in the Gauss focus and its contrast was minimized, whereas the PVP-capped Rh NSs were close to the Scherzer focus, giving a high contrast. Experimental images (see Fig. 1c) were processed using the crystallographic average method to further improve the signal-to-noise ratio (see Fig. 1c, inset). The images reveal the single-crystalline nature of Rh NS, which possesses a hexagonal structure, with a lattice parameter of $c a$. $2.6 \AA$, close to the atomic distance of $\mathrm{Rh}(2.69 \AA)$ in the (111) plane of the bulk $\mathrm{Rh}$ phase. From the viewpoint of symmetry, both face-centeredcubic (fcc) and hexagonal-close-packed (hcp) structures could give a hexagonal lattice-in the [111] and [0001] directions, respectively. In the [111] zone axis of fcc $\mathrm{Rh}$, however, the projected structure would show a lattice parameter of $1.55 \AA$, which is not the case here.

To evaluate the thickness of Rh NS, the PVP capping on the surface of Rh NSs was removed by calcinating the PVP-capped Rh NSs supported on silica wafer in flowing $\mathrm{O}_{2}$ at $600^{\circ} \mathrm{C}$, which led to the formation of silica wafer-supported $\mathrm{Rh}$ NS (see Methods for details). AFM imaging of the Rh NS supported on silica wafer shows that the sheet-like features were well preserved compared with TEM images (see Fig. 1a,b) upon above treatment. Section analysis and height profile (see Fig. 1d) reveal that the height of Rh NS is $c a$. $4 \AA$. Given the Rh atomic radius of $1.73 \AA$, this Rh NS likely consists of only single $\mathrm{Rh}$ atomic layer in thickness, which is also confirmed by HRTEM (see Supplementary Fig. 5 and Methods for details).

Analysis of the intensity profiles of electron diffraction patterns has been demonstrated to be a valid and important method for the accurate determination of the thickness of a graphene sheet ${ }^{14}$. For instance, in single-layered graphene the intensities of diffraction spots in the outer hexagons are comparable to those in the inner hexagons, whereas for multilayers the diffraction spots in the outer hexagons are more intense ${ }^{15}$. In the case of PVP-capped Rh NS, since the thickness of bare Rh NS is less than the extinction distance in electron dynamical diffraction-which is typically tens of nanometres-kinematical electron diffraction was performed.
Figure $2 \mathrm{a}$ shows a typical experimental electron diffraction pattern and the corresponding intensity profile of the PVPcapped Rh NSs. Two sets of diffraction spots obtained from two PVP-capped Rh NSs (rotated by 16 degree with respect to each other) are labelled by the full (blue) cycles and dotted (red) cycles in Fig. 2a. Diffracted intensity profiles taken along the full (blue) line denoted by (10) and (11) directions showed clearly that the intensity of the spot in the inner hexagon is twice the intensity of the spot in the outer hexagon. The same goes for the other set of diffraction spots labelled by dotted (red) cycles. For a quantitative comparison between the experimental and simulated diffraction patterns, JEMS software was used to obtain simulated diffraction patterns for candidate structures with thicknesses up to six $\mathrm{Rh}$ atomic layers (see Fig. 2b-e and Supplementary Fig. 6). The corresponding intensity profiles for the simulated diffraction patterns are also given for comparison. It is noteworthy that the intensity of the inner spot (10) is twice the intensity of the outer spot (11) only for the simulated diffraction patterns for singlelayered Rh (see Fig. 2b), which is consistent with the experimental diffraction patterns (see Fig. 2a). For the other multi-layered structures, the calculated intensities do not match the experimental pattern: for the double layered structures $(A B)$ or hcptype triple layers (ABA) the beams (10), (01) and (11) have similar intensities, and in the fcc-type triple layers (ABC) the (10) and (01) beams are absent, corresponding to an image of a hexagonal lattice with a lattice constant of $1.55 \AA$. Since the calculated patterns for lattices with more than three layers do not match the experimental pattern either, a planar single-atom layer sheet of Rh capped with PVP is indicated for the nanosheet.

Although HRTEM, AFM and electron diffraction analysis all provide evidence of an ultrathin or even single-atom layer structure of Rh NS, the conclusion is only valid for the limited number of Rh NSs studied in the microscope. Inasmuch as XAS is a powerful technique for studying the atomic structure of nanomaterials ${ }^{16}$ due to its ability in providing a statistical result for a bulk sample, it was used to elucidate the local coordination environment of $\mathrm{Rh}$ atoms and to determine the thickness of $\mathrm{Rh}$ NS on a macroscopic level (see Methods for details). For comparison the XAS spectra of Rh foil and PVP-capped 3D Rh nanoparticles ( $\mathrm{Rh}$ NPs, with an average size of $\sim 13.5 \mathrm{~nm}$, see a

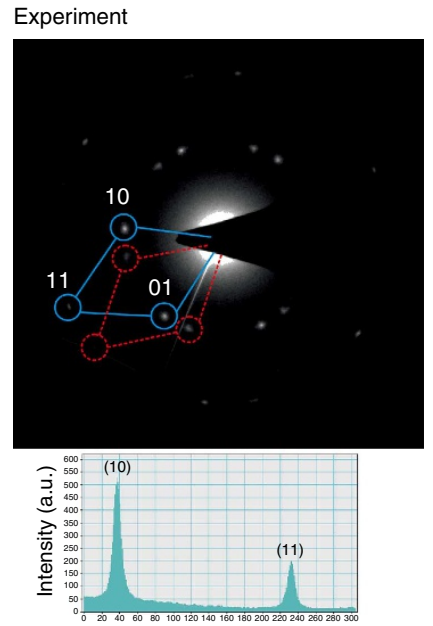

b Simulation

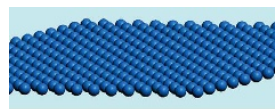
Single layer (A)
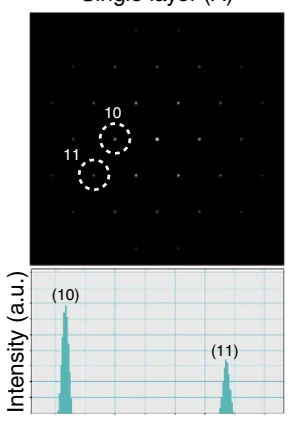

C

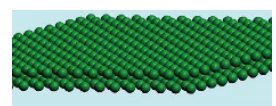

Double layers $(A B)$
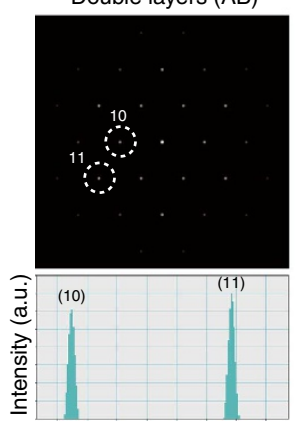

d

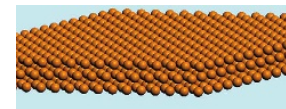

Triple layers (ABA)
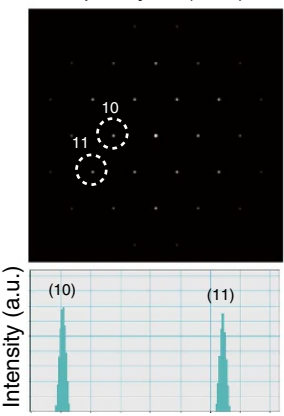

e

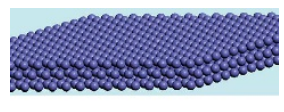

Triple layers $(A B C)$
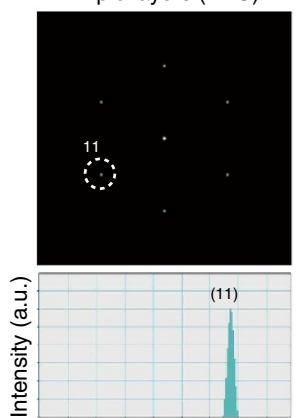

Figure 2 | Experimental and simulated electron diffraction patterns. (a) Experimental electron diffraction pattern and the corresponding intensity profiles of the PVP-capped Rh NSs. Simulated electron diffraction patterns and the corresponding intensity profiles of (b) single layer, (c) double layers, (d) triple layers in a hcp $A B A$ stacking sequence and (e) triple layers in an fcc $A B C$ stacking sequence of Rh crystals. The intensity profiles of the diffraction patterns show the relative intensities of the (10) and (11) diffraction beams. The letters ' $A$ ', ' $B$ ' and ' $C$ ' denote the relative positions of the atomic layers within the sheet plane. 

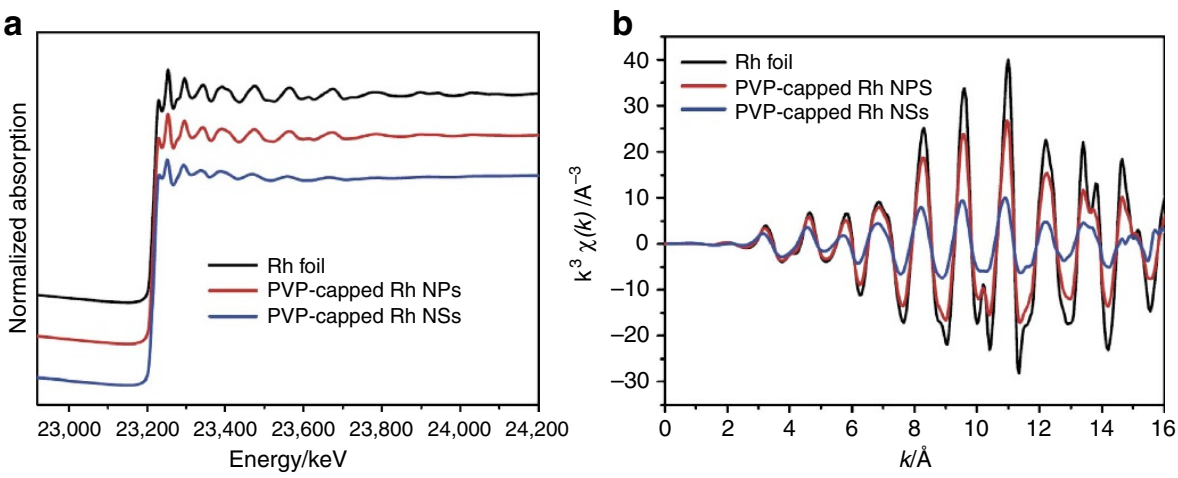

C
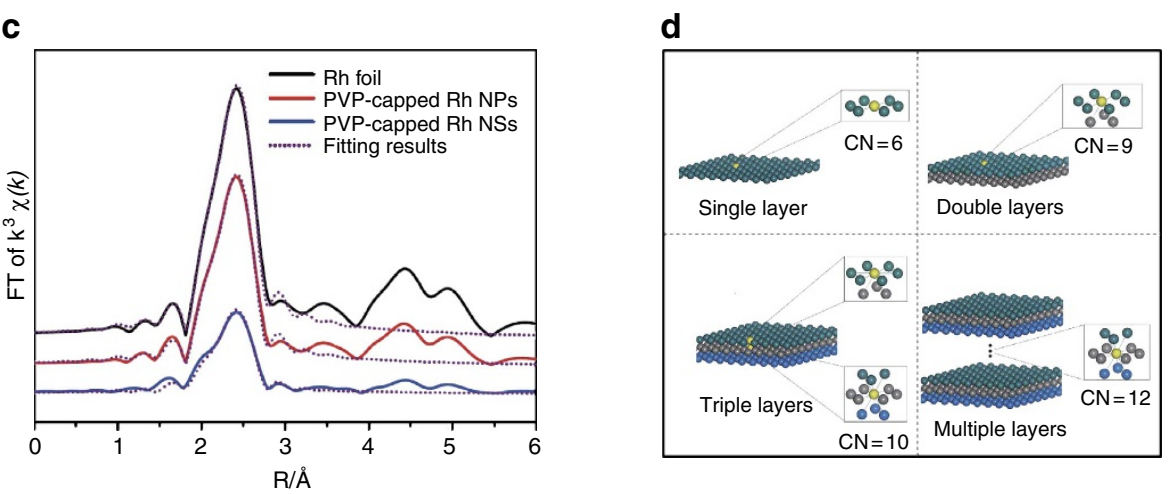

Figure 3 | Structure determination of ultrathin Rh NSs by XAS. (a) Normalized Rh K-edge EXAFS spectra of Rh foil, PVP-capped Rh NPs, and PVP-capped Rh NSs. (b) $k^{3}$-weighted Rh K-edge k-space oscillations of Rh foil, PVP-capped Rh NPs and PVP-capped Rh NSs. (c) Fourier-transformed spectra of $k^{3}$-weighted Rh K-edge EXAFS of Rh foil (solid black line), PVP-capped Rh NPs (solid red line) and PVP-capped Rh NSs (solid blue line) and corresponding curve-fitting results (dotted purple lines). (d) Schematic illustration of the coordination states with CN of Rh NS with single, double, triple and multiple layers with an exposed $\mathrm{Rh}$ (111) lattice plane (the yellow balls indicate the 'core' Rh atoms).

Table 1 | Structural parameters obtained from the curve-fitting analysis of the EXAFS spectra shown in Fig. 3.

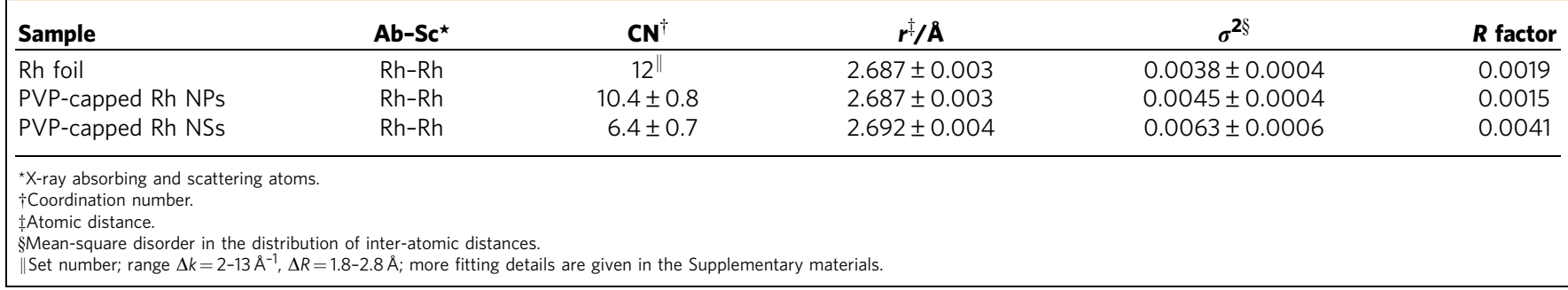

Supplementary Fig. 7 for the TEM image and Methods for synthesis) were also measured. The X-ray absorption fine structure (XAFS) spectra and k-space spectra (see Fig. 3a,b, respectively) of the PVP-capped Rh NPs and PVP-capped Rh NSs are similar and closely resemble that of Rh foil, but with reduced oscillations in the case of the PVP-capped Rh NSs. Indeed, in the Fourier-transformed extended XAFS (EXAFS) spectra (see Fig. 3c), the intensity of the peak at $2.37 \AA$, assigned to the first shell $\mathrm{Rh}-\mathrm{Rh}$ scattering, decreases in the order Rh foil $>$ PVPcapped Rh NP $\gg$ PVP-capped Rh NS. This result indicates that the $\mathrm{Rh}$ atoms in the PVP-capped Rh NSs possess a coordination environment that is different from that of the $\mathrm{Rh}$ atoms in the PVP-capped Rh NPs and foil samples. The Rh-Rh coordination number $(\mathrm{CN})$ is sensitive to both particle size and morphology at the nanoscale. The coordination environments in structures with different number of $\mathrm{Rh}$ layers are illustrated schematically in Fig. 3d. For a single-layered, close-packed, infinite Rh structure, the first shell $\mathrm{CN}$ is 6 , as each $\mathrm{Rh}$ atom is surrounded by 6 other $\mathrm{Rh}$ atoms. Double- and triple-layered Rh structures have higher
CNs (9 and 10 respectively) due to the additional inter-layer Rh$\mathrm{Rh}$ coordination. For close-packed NPs, the $\mathrm{CN}$ is also high-at least 9 for NPs with a particle size $>2 \mathrm{~nm}$ (ref. 17). From curve fitting (Table 1), the PVP-capped Rh NPs have a CN of $10.4 \pm 0.8$, in agreement with the expected value. In contrast, a $\mathrm{CN}$ of only $6.4 \pm 0.7$ is obtained for the PVP-capped Rh NSs, fitting well with the $\mathrm{CN}$ of a single-layered system. In addition, the average $\mathrm{Rh}-\mathrm{Rh}$ bond length in PVP-capped Rh NSs, estimated as $2.692 \pm 0.004 \AA$ from the XAS spectra, is similar to that of $\mathrm{Rh}$ foil $(2.687 \pm 0.003 \AA)$, indicating the Rh NS is composed of closepacked $\mathrm{Rh}$ atoms.

In addition, we analysed the EXAFS data by taking into account the Rh-O contribution. Listed in Supplementary Fig. 8 and Supplementary Table 1 are the Fourier-transformed EXAFS spectra of Rh NS in the range of 2-13 inverse Angstroms, and the fitting results with and without considering a $\mathrm{Rh}-\mathrm{O}$ shell. We also present in Supplementary Fig. 9 and Supplementary Table 2 the Fourier-transformed EXAFS spectra of Rh foil and Rh NS in the range of 2-8 inverse Angstroms and their corresponding fitting 
results. Clearly incorporating a $\mathrm{Rh}-\mathrm{O}$ shell significantly improved the fitting quality, when the k-range is reduced to 2-8 inverse Angstroms, suggesting that the $\mathrm{Rh}-\mathrm{O}$ bonds plausibly exist on $\mathrm{Rh}$ nanosheets. In this case, the $\mathrm{CN}$ of $\mathrm{Rh}-\mathrm{Rh}$ is $5.6 \pm 1.0$, essentially the same as the single $\mathrm{Rh}-\mathrm{Rh}$ shell fitting results presented in our work. The $\mathrm{CN}$ of $\mathrm{Rh}-\mathrm{O}$ is $1.0 \pm 0.6$. Here the large uncertainty is simply because the number of independent fitting parameters exceeds the statistic limitation.

Electronic structures and stability. In order to elucidate the nature of the unexpected stability of the prepared single-layered Rh NSs, DFT studies (see Methods for details) were carried out on finite planar $\mathrm{Rh}_{x}(x=7,19,37,61)$ clusters with $D_{6 \mathrm{~h}}$ pointgroup symmetry (see Supplementary Fig. 10) and an infinite single-layered slab. Figure 4a compares the bonding pattern of $\mathrm{C}$ atom in graphene and $\mathrm{Rh}$ atom in Rh NSs. The calculated band structures and local density of states for the single-layered slab model are shown in Fig. 4b. Our calculated spin densities on the central atoms of the clusters and the magnetic moment in the periodic slab are close to 1 for each $\mathrm{Rh}$ atom. The calculated nucleus-independent chemical shift value at $1 \AA$ above the centre of the $\mathrm{Rh}_{7}$ hexagon is -12.04 p.p.m., which is close to that for
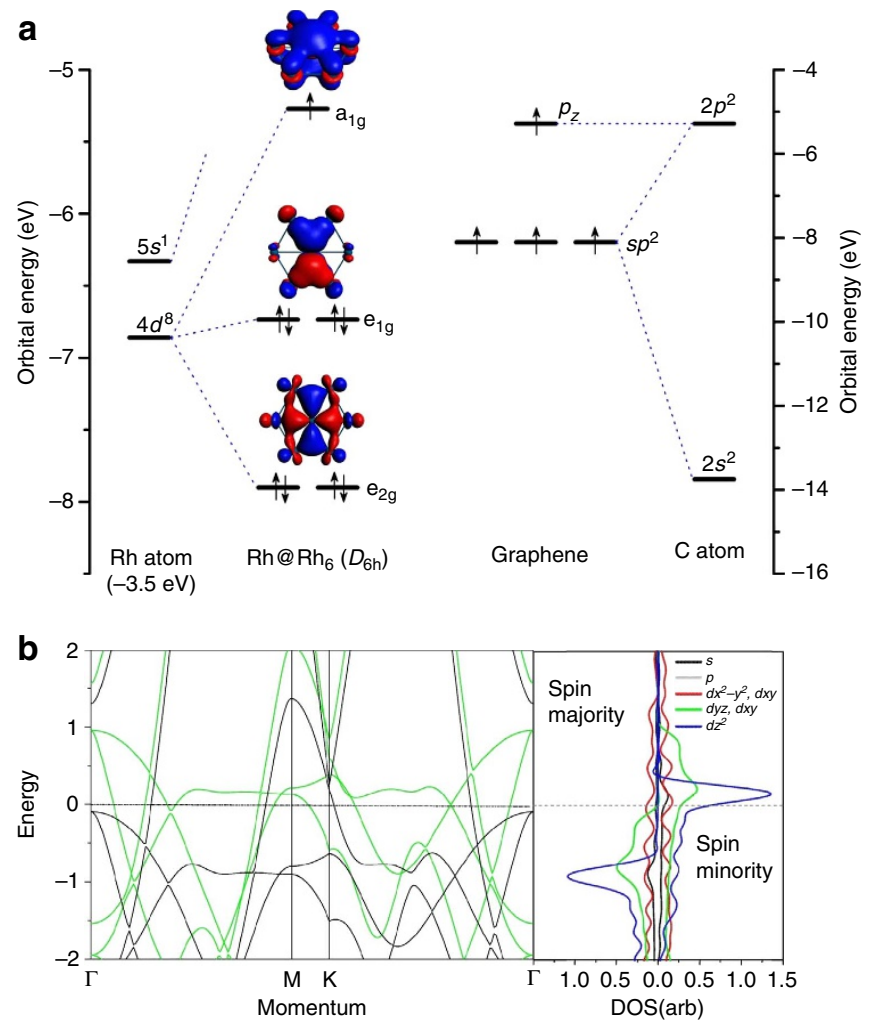

Figure 4 | DFT calculations of single-layered structure of Rh atoms. (a) Rh $4 d$ orbitals split into $e_{2 g}\left(d_{x y}, d_{x^{2}-y^{2}}\right), e_{1 g}\left(d_{x z}, d_{y z}\right)$ and $a_{1 g}\left(d_{z^{2}}\right)$ species upon interacting with six peripheral $R h$ atoms in $a \mathrm{Rh}_{7}\left(\mathrm{D}_{6 \mathrm{~h}}\right)$ cluster (left). The highest $\mathrm{a}_{1 \mathrm{~g}}$ orbital forms the delocalized $\delta$-bonding mentioned in the text. The Kohn-Sham orbitals are plotted with isosurface value $=0.03$ atomic units. The energy levels of the central Rh atom in a vacuum are shifted by $-3.5 \mathrm{eV}$ for clarity. C $\left(2 s^{2} 2 p^{2}\right)$ forms three $s p^{2}$ hybrid orbitals and one $p_{z}$ orbital during the formation of graphene (right). (b) The band structures (left) and the local spin-orbital density of states (LDOS) (right) of a single-layered Rh NS-derived from a periodic slab model. The black and green lines in the band structures correspond to the spin-majority and spin-minority bands, respectively. The $\sigma-, \pi$ - and $\delta$-contributions are shown in red, green and blue colours in the LDOS, respectively. benzene ( -11.2 p.p.m.) and other polybenzenoid hydrocarbons of graphene fragments ${ }^{18}$.

Catalytic properties. Hydrogenation ${ }^{19}$ and hydroformylation ${ }^{20}$ reactions represent important industrial processes, producing key feedstocks for the synthesis of fine chemicals. The commonly used catalyst for these reactions is $\mathrm{Rh}$ metal ${ }^{21,22}$. However, given its limited reserves and high price compared with other noble metals, it is important to design Rh catalysts with high atomic efficiency. One way of doing this is to fabricate single-layer materials with $100 \%$ exposed $\mathrm{Rh}$ atoms with unsaturated coordination. To investigate the catalytic properties of PVPcapped Rh NSs, hydrogenation of phenol and hydroformylation of 1-octene were employed as probe reactions. Rh NPs and commercial Rh/C (see Supplementary Figs 6 and 11, respectively) were chosen as the benchmark catalysts (see Methods for details). Figure 5a displays a comparison of the conversion of phenol over reaction time catalysed by different $\mathrm{Rh}$ catalysts. The best performance was achieved with PVP-capped Rh NSs, which gave $>99.9 \%$ conversion within $4 \mathrm{~h}$ at near room temperature $\left(30^{\circ} \mathrm{C}\right)$ under low $\mathrm{H}_{2}$ pressure (1.0 MPa) and showed an activity that was, respectively, four- and seven times greater than those of commercial Rh/C and PVP-capped Rh NPs (based on the conversion at $1 \mathrm{~h}$ ). Without further optimization, all the catalysts show comparable selectivity to cyclohexanone and cyclohexanol (see Supplementary Table 3). Especially, in the hydroformylation of 1-octene, when compared with commercial $\mathrm{Rh} / \mathrm{C}$ and PVPcapped Rh NPs, our PVP-capped Rh NSs was found to give both superior catalytic activity and selectivity towards the target product (aldehyde) under mild reaction conditions (see Fig. 5b and Supplementary Table 4). Since surface atoms are important in heterogeneous catalysis ${ }^{23}$, a high proportion of surface atoms will enhance the efficiency of catalytic reactions. The proportion of surface Rh atom in the PVP-capped Rh NSs is $100 \%$, whereas this is not possible in the commercial $\mathrm{Rh} / \mathrm{C}$ or PVP-capped $\mathrm{Rh}$ NPs (see Supplementary Figs 12 and 13, Supplementary Table 5 and Supplementary Note 1 for more details). As a result, a full exposure of Rh atoms in the PVP-capped Rh NSs accounts for the largely enhanced catalytic activity.

\section{Discussion}

We have successfully synthesized single-layered Rh NSs capped with PVP. HRTEM, AFM, electron diffraction analysis and XAS studies suggest that the Rh NSs contain single-atom layer of Rh. It is not unreasonable to expect that our synthetic approach might be extended to the preparation of surfactant-capped single-atomlayered sheets of other metals. These single-layered NSs of metals might find potential applications in catalysis and material physics.

Metals with an fcc or hcp structure have non-directional 3D metallic bonding, rather different from layer-structured materials such as graphene with strong in-plane $\sigma$-bonding together with $\pi$-bonding by the perpendicular orbitals ${ }^{2}$. As shown in Fig. $4 \mathrm{a}$, in graphene each $\mathrm{C}$ atom has half-filled $\left(s p^{2}\right)^{3}$ hybrid orbitals that form the $\sigma$-skeleton and a $\left(p_{z}\right)^{1}$ orbital that form the familiar delocalized $\pi$-bonding framework. Our calculation show that in the single-layered $\mathrm{Rh}$ sheet each $\mathrm{Rh}$ atom has fully filled $\left(d_{x y}\right.$, $\left.d_{x^{2}-y^{2}}\right)^{4}$ and $\left(d_{x z}, d_{y z}\right)^{4}$ orbitals available for weak $\sigma$ - and $\pi$-type interactions with surrounding Rh atoms, and a half-filled $\left(d_{z^{2}}\right)^{1}$ for shoulder-to-shoulder $\delta$-type interactions with other $\mathrm{Rh}$ centres $^{24}$. These results agree with our calculated spin densities on the central atoms of the clusters and the magnetic moment in the periodic slab. As a result of the significant $d_{z^{2}}-s$ orbital mixing, a conjugated $\delta$-bonding framework composed of the $d_{z^{2}}-s$ hybrid orbitals of the Rh atoms is generated, which is responsible for electron delocalization throughout the whole 

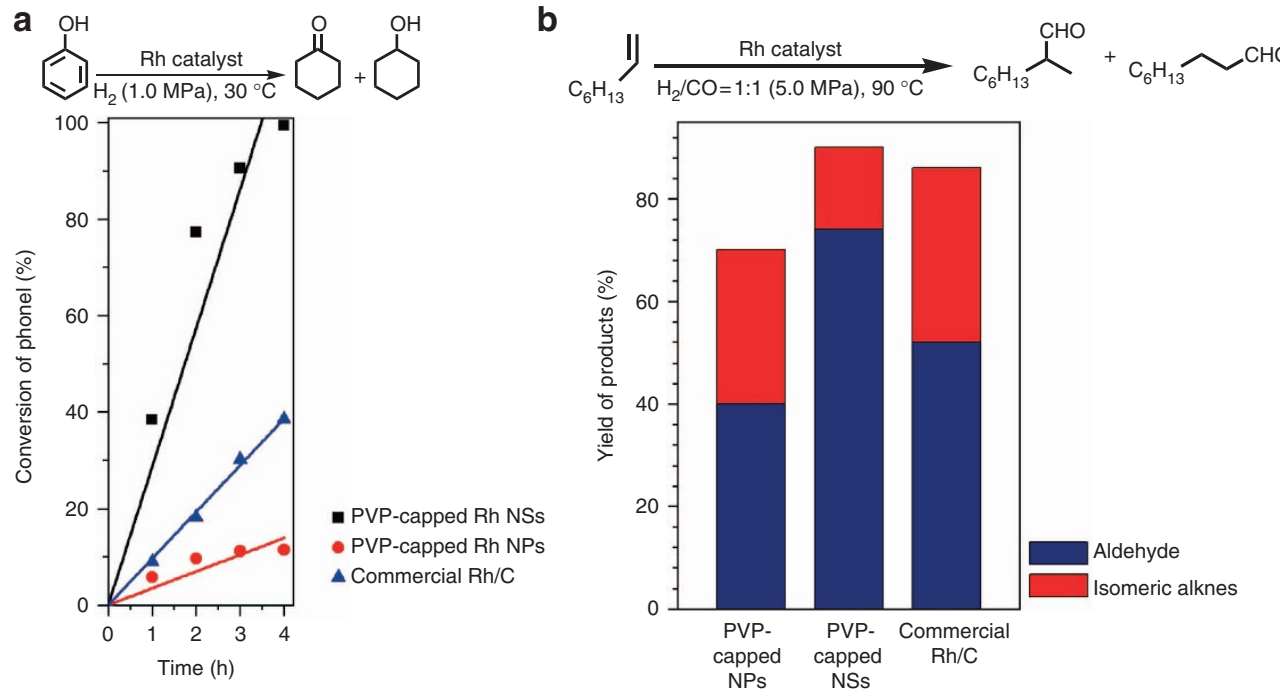

Figure 5 | Comparison of catalytic ability of PVP-capped Rh NSs, PVP-capped Rh NPs, and commercial Rh/C. (a) Hydrogenation of phenol and (b) hydroformylation of 1-octene. Reaction conditions and other details are shown in Supplementary Tables 3 and 4.

cluster (see Fig. 4a). The calculated band structures and local density of states (see Fig. $4 \mathrm{~b}$ ) for the single-layered slab model also show that in the single-layered $\mathrm{Rh}$ sheet the bonding and antibonding $\sigma$ - and $\pi$-interactions nearly cancel out, while the $4 d_{z^{2}}$ (and 5s) orbitals from all the Rh centres form spin-polarized bands with a net bonding effect, which contribute to the high surface energy and electron delocalization.

Clearly the $\delta$-bonding delocalized in the single-layered $\mathrm{Rh}$ sheet contributes to its stability. The calculated nucleusindependent chemical shift value indicates that the single-layered $\mathrm{Rh}$ sheet has quasi-aromatic stability and a diatropic ring current in a magnetic field. On the other hand, the formation of conjugated $\delta$-bonding partially saturates the dangling bonds, thereby to a certain extent decreasing the surface energy of the single-layered Rh. Despite the conjugated $\delta$-bonding contribution to the stability of the Rh sheet, a bare Rh sheet is likely less stable than a 3D packed structure, thus requiring further stabilization through surface ligands such as $\mathrm{PVP}^{25}$. Indeed, while the calculated phonon spectrum of the single-layered Rh NSs show non-negligible imaginary frequencies for the out-of-plane motions of Rh atoms (see Supplementary Fig. 14a), these imaginary frequency modes are largely suppressed upon adsorption of simplified PVP ligands (2-pyrrolidone molecule), with the imaginary frequencies significantly reduced to within those of the numerical noise (see Supplementary Fig. 14b). Moreover, the surface energy decreases from 0.126 for bare $\mathrm{Rh}$ sheet to $0.071 \mathrm{eV}^{-2}$ for the simplified PVP-covered ones. These results are in line with the experimental observation that the single-layered $\mathrm{Rh}$ NSs are formed with the help of PVP capping. Consistent with these theoretical results, our experiment shows that fabrication in the absence of PVP yields aggregates of Rh NSs (see Supplementary Fig. 15). Because of the unique bonding of the $\mathrm{Rh}$ atoms, the resulting nanoscale materials may find applications in electronics, magnetic devices and catalysis. Based on the analogy with graphene, we propose to call the single-layered metal nanosheet as 'metallene'.

\section{Methods}

Reagents. $\mathrm{Rh}(\mathrm{III})$ acetylacetonate $\left(\mathrm{Rh}(\mathrm{acac})_{3}, 99 \%\right)$, commercial $\mathrm{Rh}$ black, commercial $\mathrm{Rh} / \mathrm{C}$ (5\% metal) were purchased from Alfa Aesar. Poly(vinylpyrrolidone) (PVP, MW $=30,000)$, L-ascorbic acid (AA), benzyl alcohol, formaldehyde, phenol, cyclohexanone, cyclohexanol and 1-octene were purchased from Sinopharm Chemical Reagent Co. Ltd. (Shanghai, China). All regents were used as received without further purification.

Synthesis of PVP-capped ultrathin Rh nanosheets. $\mathrm{Rh}(\mathrm{acac})_{3}(8.0 \mathrm{mg})$, PVP $(\mathrm{MW}=30,000,120.0 \mathrm{mg})$ were dissolved in benzyl alcohol $(3 \mathrm{ml})$ and formaldehyde $(3 \mathrm{ml})$. The mixture was stirred vigorously for $1 \mathrm{~h}$ and then transferred to a Teflon-lined stainless steel autoclave. The autoclave was sealed and maintained at $180^{\circ} \mathrm{C}$ for $8 \mathrm{~h}$ and then cooled to room temperature. The resulting black product was precipitated with acetone $(10 \mathrm{ml})$, separated in a centrifuge and washed three times with ethanol $(10 \mathrm{ml})$ and finally dried under vacuum.

Synthesis of PVP-capped Rh nanoparticles. $\mathrm{Rh}(\mathrm{acac})_{3}(8.0 \mathrm{mg}), \mathrm{PVP}$ $(\mathrm{MW}=30,000,120.0 \mathrm{mg})$ and AA $(40 \mathrm{mg})$ were dissolved in benzyl alcohol $(6 \mathrm{ml})$. The mixture was stirred vigorously for $\sim 1 \mathrm{~h}$ and then transferred to a Teflon-lined stainless steel autoclave. The autoclave was sealed and maintained at $180^{\circ} \mathrm{C} \mathrm{for} 8 \mathrm{~h}$ and then cooled to room temperature. The resulting black product was precipitated with acetone $(10 \mathrm{ml})$, separated in a centrifuge and washed three times with ethanol $(10 \mathrm{ml})$ and finally dried under vacuum.

Transmission electron microscope analysis. Transmission electron microscope (TEM) and HRTEM studies were performed on a Hitachi model H-800 TEM or JEOL-2010F HRTEM operating at $200 \mathrm{kV}$.

HRTEM analysis. HRTEM images were recorded on a JEOL JEM-2100 microscope using bright-field microscopy at an accelerating voltage of $200 \mathrm{kV}$.

Aberration-corrected electronic microscopy analysis. Aberration-corrected electronic microscopy observations were performed on an FEI Titan 80-300 TEM equipped with a spherical aberration (Cs) corrector as the objective lens. The information limit of the microscope is $0.08 \mathrm{~nm}$. The images were taken using the negative-Cs imaging technique, which was shown to give high contrast and low noise. The images were taken at a high tension of $300 \mathrm{kV}$, with the spherical aberration set at around $-13 \mu \mathrm{m}$. Because of the high sensitivity of the PVP-capped $\mathrm{Rh}$ NSs to irradiation by the electron beam, the electron exposure was kept as low as possible during the experiments. Kinematical electron diffraction patterns were simulated using MacTempas and JEMS softwares. For the PVP-capped Rh NSs, dynamic diffraction was neglected.

AFM analysis. AFM analysis was performed in tapping mode in air with a Multimode Nanoscope IIIa SPA (Veeco Instruments, Bruker). Ultrasharp cantilevers with a diamond-like carbon tip (NSG01, NT-MDT, Russia) were used. The image was flattened using the NanoScope Analysis software (version 1.40). A silicon wafer with thermal oxide layer of $2.850 \AA$ in thickness (Silicon Valley Microelectronics, Inc. USA) was used.

The surfactant PVP could be removed after high temperature oxygen pretreatment reactions, as reported by Somorjai et al. ${ }^{26-28}$ In our study, The AFM 
sample was prepared by the following procedures: PVP-capped Rh NSs was initially dispersed in ethanol and then placed dropwise on the silicon wafer. The formed silica wafer supported sample was dried in air and subsequently calcinated in tubular furnace in flowing $\mathrm{O}_{2}$ from room temperature to $600^{\circ} \mathrm{C}$ with a heating rate of $0.5^{\circ} \mathrm{C} \mathrm{min}^{-1}$, after which the temperature was maintained at $600^{\circ} \mathrm{C}$ for $1 \mathrm{~h}$. After this silica wafer-supported sample was cooled down to room temperature, it was treated in flowing $\mathrm{H}_{2}$ at $600^{\circ} \mathrm{C}$ for $2 \mathrm{~h}$. After these procedures, the PVP surfactant was removed by the oxidation of $\mathrm{O}_{2}$, leaving Rh NSs supported on silica wafer, which was then used for the AFM analysis.

Observation of cross-section of Rh nanosheets. The PVP-capped Rh NSs were dispersed in $1 \mathrm{ml}$ of epoxy resin embedding medium (Epon 812). After 15-min ultrasonic re-dispersion, a drop of 2,4,6-Tris(dimethylaminomethyl)phenol (DMP-30) was added as a hardener. Subsequently, the mixture was dried at $80^{\circ} \mathrm{C}$ in an oven for $24 \mathrm{~h}$. Ultrathin sections ( $\sim 30 \mathrm{~nm}$ thickness) were prepared using a Diatome diamond knife on a Leica EM UC6 ultramicrotome, and transferred onto $\mathrm{Cu}$ grids with a lacey carbon supporting film. The embedding medium stabilized the Rh NSs against electron irradiation and simultaneously fixed the Rh NSs in the hard mixture in random directions. By using this method, the morphology of ultrathin Rh NSs was maintained under electron irradiation to some extent and the cross-section images could be observed. Supplementary Fig. 5 shows the crosssection of an assembly of Rh NSs embedded in an epoxy resin, in which three Rh NSs (shown by arrows) were found to pack parallel to each other with a distance of ca. $7.6 \AA$, forming a lamellar structure. Each sheet has a thickness of $\sim 3.6 \AA$, an order of magnitude smaller than the thickness of most reported ultrathin metallic nanosheets.

XAS analysis. XAS experiments were performed at Photon Factory, KEK, Tsukuba, Japan, under the approval of the Photon Factory Advisory Committee (proposal no. 2012G601). The X-ray energy was calibrated using an inflection point of Rh foil in the X-ray absorption near edge structure. The XAS measurements of the Rh K-edge of PVP-capped Rh NSs and PVP-capped Rh NPs dispersed in ethanol $\left(\mathrm{Rh}=c a .10 \mathrm{mg} \mathrm{ml}^{-1}\right)$ were carried out at room temperature in transmission mode, by employing a $\mathrm{Si}(311)$ crystal for the monochromatic synchrotron radiation. Data reduction of the XAFS spectra was conducted with Athena and Artemis included in the Ifeffit package. The $\mathrm{k}^{3}$-weighted EXAFS oscillation in the range $2-13 \AA^{-1}$ was Fourier-transformed and curve-fitting analysis was performed in the range $1.8-2.8 \AA$ in $\mathrm{R}$ space. For curve-fitting analysis, the backscattering factor and the phase shift of the scattering path were calculated with FEFF6L using the crystal structure of $\mathrm{Rh}$ metal. The amplitude reduction factor was also estimated by fitting the spectrum of Rh foil with the parameters calculated with FEFF.

Details of theoretical calculations. Cluster models: DFT calculations were carried out on a series of planar $\mathrm{Rh}_{x}(x=7,19,37,61)$ clusters with $D_{6 \mathrm{~h}}$ point-group symmetry (see Supplementary Fig. 10) by using the Amsterdam Density Functional code (ADF 2010.02). ${ }^{29-31}$ The PBE functional ${ }^{32}$ and a Slater-type-orbital (STO) basis sets with quality of double- $\zeta(\mathrm{DZ})$ and triple- $\zeta$ plus polarization functions $(\mathrm{TZP})^{33}$ were used for all atoms. The frozen core approximation was employed for inner shells $\left[1 s^{2}-3 d^{10}\right]$ of Rh. Numerous calculations were performed to determine the spin multiplicities of these clusters. The smallest $\mathrm{Rh}_{7}$ cluster was found to have 13 unpaired electrons, with roughly two spins on the peripheral $\mathrm{Rh}$ atoms and one spin on the central $\mathrm{Rh}$ atom. Figure $4 \mathrm{a}$ shows the orbital splittings of $\mathrm{C}$ in graphene as $s p^{2}+p_{z}$ and $\mathrm{Rh}$ in the $\mathrm{Rh}_{7}$ sheet as $\sigma\left(d_{x y}, d_{x^{2}-y^{2}}\right), \pi\left(d_{x z}, d_{y z}\right)$ and $\delta\left(d_{z^{2}}\right)$ species, which are mainly composed of $4 \mathrm{~d}$ orbitals of the central Rh atom. The central Rh atom of the $\mathrm{Rh}_{7}$ cluster tends to have similar properties to those of an arbitrary atom of a single-layered $\mathrm{Rh}(111)$ surface. Owing to the ligand field in the $D_{6 \mathrm{~h}}$ point group, the $d$ orbitals of the central Rh atom split into three groups with irreducible representations $e_{2 g}, e_{1 g}$ and $a_{1 g}$, mainly participating in the corresponding molecular orbitals displayed in Fig. 4a. Note that the highest $\mathrm{a}_{1 \mathrm{~g}}$-type orbitals from each $\mathrm{Rh}$ atom form the delocalized $\delta$-bonding mentioned in the text, which involves the $d_{z^{2}}-s$ hybrid orbital of Rh atoms.

Slab model for single-layered Rh. The periodic DFT calculations were performed using the Vienna $a b$ initio simulation package (VASP) ${ }^{34}$. The spin-polarized generalized gradient approximation with the PBE functional ${ }^{32}$ and the projector augmented wave (PAW) method ${ }^{35}$ with a cutoff energy of $400 \mathrm{eV}$ were used. A $p(3 \times 3)$ supercell was adopted for single-layered $\mathrm{Rh}$ with the vacuum spacing set at least $28 \AA$. Integration over the Brillouin zone was achieved using the Monkhorst-Pack scheme with $11 \times 11 \times 1$ and $33 \times 33 \times 1$ meshes for structural optimizations and electronic structure calculations, respectively. The phonon spectra were calculated on a Rh nanosheet capped with simplified PVP ligands (2-pyrrolidone molecules) within the usual harmonic approximation.

Procedure for hydrogenation of phenol. In a typical catalytic reaction, phenol (0.1 $\mathrm{mmol}), \mathrm{ZnCl}_{2}(0.01 \mathrm{mmol}), 0.64 \mathrm{~mol} \% \mathrm{Rh}$ catalysts (PVP-capped Rh NSs, PVP-capped Rh NPs or commercial $\mathrm{Rh} / \mathrm{C}$ ) suspension in $\mathrm{CH}_{2} \mathrm{Cl}_{2}(1 \mathrm{ml})$ were placed in an autoclave. To remove the air from the reaction system, the autoclave was purged three times with $\mathrm{H}_{2}$. Subsequently, the autoclave was pressurized with
$\mathrm{H}_{2}(1.0 \mathrm{MPa})$ and the reaction mixture was stirred at 600 r.p.m. at $30^{\circ} \mathrm{C}$ for the required time.

Procedure for the hydroformylation of 1-octene. In a typical catalytic reaction, 1 -octene $(5.0 \mathrm{mmol})$ and $0.02 \mathrm{~mol} \% \mathrm{Rh}$ catalysts (PVP-capped Rh NSs, $\mathrm{PVP}$-capped $\mathrm{Rh} \mathrm{NPs}$ or commercial $\mathrm{Rh} / \mathrm{C})$ suspension in isopropanol $(15 \mathrm{ml})$ were placed in an autoclave. To remove the air from the reaction system, the autoclave was purged three times with $\mathrm{N}_{2}$ and then three times with $\mathrm{H}_{2} / \mathrm{CO}$ (1:1) mixed gas. Subsequently, the autoclave was pressurized with $\mathrm{H}_{2} / \mathrm{CO}(1: 1,5.0 \mathrm{MPa})$ mixed gas and the reaction mixture was stirred at 580 r.p.m. at $90^{\circ} \mathrm{C}$ for $3 \mathrm{~h}$.

\section{References}

1. Novoselov, K. S. et al. Electric field effect in atomically thin carbon films. Science 306, 666-669 (2004).

2. Novoselov, K. S. et al. Two-dimensional atomic crystals. Proc. Natl. Acad. Sci. USA 102, 10451-10453 (2005).

3. He, H. et al. Magnetic resonant mode in the single-layer high-temperature superconductor $\mathrm{Tl}_{2} \mathrm{Ba}_{2} \mathrm{CuO}_{6}+\delta$. Science 295, 1045-1047 (2002).

4. Novoselov, K. S. et al. Two-dimensional gas of massless Dirac fermions in graphene. Nature 438, 197-200 (2005).

5. Zhang, Y. B., Tan, Y. W., Stormer, H. L. \& Kim, P. Experimental observation of the quantum Hall effect and Berry's phase in graphene. Nature 438, 201-204 (2005).

6. Wang, X. et al. Room-temperature all-semiconducting sub-10-nm graphene nanoribbon field-effect transistors. Phys. Rev. Lett. 100, 206803 (2008).

7. Geim, A. K. Graphene: status and prospects. Science 324, 1530-1534 (2009).

8. Balandin, A. A. Thermal properties of graphene and nanostructured carbon materials. Nat. Mater. 10, 569-581 (2011).

9. Chhowalla, M. et al. The chemistry of two-dimensional layered transition metal dichalcogenide nanosheets. Nat. Chem. 5, 263-275 (2013).

10. Huang, X. Q. et al. Freestanding palladium nanosheets with plasmonic and catalytic properties. Nat. Nanotechnol. 6, 28-32 (2011).

11. Jang, K., Kim, H. J. \& Son, S. U. Low-Temperature synthesis of ultrathin rhodium nanoplates via molecular orbital symmetry interaction between rhodium precursors. Chem. Mater. 22, 1273-1275 (2010).

12. Yin, A. X. et al. Ru nanocrystals with shape-dependent surface-enhanced raman spectra and catalytic properties: controlled synthesis and DFT calculations. J. Am. Chem. Soc. 134, 20479-20489 (2012).

13. Yu, R., Lentzen, M. \& Zhu, J. Effective object planes for aberration-corrected transmission electron microscopy. Ultramicroscopy 112, 15-21 (2012).

14. Dato, A., Radmilovic, V., Lee, Z., Phillips, J. \& Frenklach, M. Substrate-free gas-phase synthesis of graphene sheets. Nano Lett. 8, 2012-2016 (2008).

15. Hernandez, Y. et al. High-yield production of graphene by liquid-phase exfoliation of graphite. Nat. Nanotechnol. 3, 563-568 (2008).

16. Billinge, S. J. L. \& Levin, I. The problem with determining atomic structure at the nanoscale. Science 316, 561-565 (2007).

17. Beale, A. M. \& Weckhuysen, B. M. EXAFS as a tool to interrogate the size and shape of mono and bimetallic catalyst nanoparticles. Phys. Chem. Chem. Phys. 12, 5562-5574 (2010).

18. Moran, D., Stahl, F., Bettinger, H. F., Schaefer, III H. F. \& Schleyer, P. v. R. Towards graphite: magnetic properties of large polybenzenoid hydrocarbons. J. Am. Chem. Soc. 125, 6746-6752 (2003).

19. Liu, H. Z., Jiang, T., Han, B. X., Liang, S. G. \& Zhou, Y. X. Selective phenol hydrogenation to cyclohexanone over a dual supported Pd-Lewis acid catalyst. Science 326, 1250-1252 (2009).

20. Franke, R., Selent, D. \& Börner, A. Applied hydroformylation. Chem. Rev. 112, 5675-5732 (2012).

21. Guerrero, M. et al. About the use of rhodium nanoparticles in hydrogenation and hydroformylation reactions. Curr. Org. Chem. 17, 364-399 (2013).

22. Yuan, Y., Yan, N. \& Dyson, P. J. Advances in the rational design of rhodium nanoparticle catalysts: control via manipulation of the nanoparticle core and stabilizer. ACS Catal. 2, 1057-1069 (2012).

23. Somorjai, G. A. \& Li, Y. Introduction to Surface Chemistry and Catalysis. 2nd edn (ed. John Wiley \& Sons, 2010).

24. Zhai, H. J., Averkiev, B. B., Zubarev, D. Y., Wang, L.-S. \& Boldyrev, A. I. $\delta$ aromaticity in $\left[\mathrm{Ta}_{3} \mathrm{O}_{3}\right]^{-}$. Angew Chem. Int. Ed. 46, 4277-4280 (2007).

25. Hoffmann, R. Small but strong lessons from chemistry for nanoscience. Angew. Chem. Int. Ed. 52, 93-103 (2013).

26. Rioux, R. M., Song, H., Hoefelmeyer, J. D., Yang, P. D. \& Somorjai, G. A High-surface-area catalyst design: synthesis, characterization, and reaction studies of platinum nanoparticles in mesoporous SBA-15 silica. J. Phys. Chem. B 109, 2192-2202 (2005).

27. Song, H. et al. Hydrothermal growth of mesoporous SBA-15 silica in the presence of pvp-stabilized pt nanoparticles: synthesis, characterization, and catalytic properties. J. Am. Chem. Soc. 128, 3027-3037 (2006).

28. Michael, E. G., Song, H., Zhang, Y. W. \& Somorjai, G. A. Colloidally synthesized monodisperse $\mathrm{Rh}$ nanoparticles supported on SBA-15 for size- 
and pretreatment-dependent studies of CO oxidation. J. Phys. Chem. C 113, 8616-8623 (2009).

29. ADF 2010.02, SCM, Vrije Universiteit, Amsterdam, The Netherlands http:// www.scm.com.

30. Guerra, C. F., Snijders, J. G., te Velde, G. \& Baerends, E. J. Towards an order-N DFT method. Theoret. Chem. Acc 99, 391-403 (1998).

31. te Velde, G. et al. Chemistry with ADF. J. Comput. Chem. 22, 931-967 (2001).

32. Perdew, J. P., Burke, K. \& Ernzerhof, M. Generalized gradient approximation made simple. Phys. Rev. Lett. 77, 3865-3868 (1996).

33. van Lenthe, E. \& Baerends, E. J. Optimized Slater-type basis sets for the elements 1-118. J. Comput. Chem. 24, 1142-1156 (2003).

34. Kresse, G. \& Furthmüller Efficient iterative schemes for ab initio total-energy calculations using a plane-wave basis set. J. Phys. Rev. B 54, 11169-11186 (1996).

35. Blöchl, P. E. Projector augmented-wave method. Phys. Rev. B 50, 17953-17979 (1994).

\section{Acknowledgements}

This work is supported by the State Key Project of Fundamental Research for Nanoscience and Nanotechnology (2011CB932401, 2011CBA00500, 2012CB224802 and 2011CB606406) and the National Natural Science Foundation of China (NSFC) (grant nos 21221062, 21131004, 21171105, 21390393, 21322107, 91026003 and 51071092). The calculations were performed using the DeepComp 7000 computer at the Supercomputer Center of the Computer Network Information Center, Chinese Academy of Sciences and the Shanghai Supercomputing Center. This work made use of the resources of the Beijing National Center for Electron Microscopy. We thank L.N. Zhang and Z.H. Han for help with the HRTEM measurements, Y.M. Zhu and Q. Zhan for electron diffraction simulations, M.F. Shao, X.W. Wang, X.H. Qiu, L.W.Chen and S.L. Xu for their help with AFM measurements, S. Hong for his advice and help in embedding and sectioning of samples, R.J. Wang for his helpful suggestion with the X-ray diffraction analysis, C. Hou, M.R. Ren and J.L. Chen for their help in the catalytic measurement. We are grateful to M Wei, X.M. Sun and D. Evans for their help with reading the manuscript, Dr. Horiuchi (Osaka Prefecture University), Mr. Kato and Dr. Nitta (JASRI) for their assistance with XAS experiments, S.Q. Wei for his valuable suggestion with the XAS analysis and S. Zhang for his help with XAS measurement.

\section{Author contributions}

H.D. conceived the idea, designed and performed the experiment, analysed the results and wrote the manuscript. N.Y. performed the XAFS measurement and data analysis. R.Y. performed aberration-corrected TEM imaging, electron diffraction analysis, and the corresponding simulations. C.-R.C., G.Z. and H.-S.H. conducted the DFT calculations. H.R. and J.M. assisted with sample synthesis and TEM characterization. Z.N. assisted in writing the abstract section and finalized the images for the paper. A.H. and T.T. performed the XAFS measurement. P.J.D. reviewed the manuscript. J.L. and Y.L. supervised the project, helped design the experiments and calculations, analysed the data and wrote the manuscript. All the authors discussed the results and commented on the manuscript.

\section{Additional information}

Supplementary Information accompanies this paper at http://www.nature.com/ naturecommunications

Competing financial interests: The authors declare no competing financial interests.

Reprints and permission information is available online at http://npg.nature.com/ reprintsandpermissions/

How to cite this article: Duan, H. et al. Ultrathin rhodium nanosheets . Nat. Commun 5:3093 doi: 10.1038/ncomms4093 (2014). 